\title{
THE FORMATION OF PROJECTED VISUAL IMAGES BY INTERMITTENT RETINAL STIMULATION.
}

\section{GENERAL CHARACTERISTICS OF THE IMAGE.}

\author{
By GEORGE H. MILES.
}

I. Introduction.

II. Method of producing the projected image: observations of its chief characteristics.

III. Part played by intermittent stimulation.

IV. Observations concerning the projection of the image.

V. Suggested explanations.

\section{INTRODUCTION.}

IT is a well-known fact that when the eyes are closed after a bright light has been fixated, an after-image of the object can be seen. A similar image can also be seen if the eyes are directed to a uniformly illuminated background, immedia,tely after the fixation. These images gradually fade; but if light is allowed to fall intermittently at a suitable rate on the closed eyelids, or if the intensity of the illumination of the screen on which the image is projected be varied at a regular rate, the following changes are observed. In each case, the fading and indistinct image is rapidly revived; it becomes clearer in outline; its intensity and objective character are strikingly increased ; its duration is prolonged ; and in many ways the image thus aroused forms such a marked contrast to the ordinary image that further investigation of its peculiarities is demanded. An attempt has been made to discover the main factors involved in the production of the projected image. In this paper a brief account is given of a few of the experiments that have been carried out with this aim in view. 
Even in the simplest experiments it was evident that, at a certain rate of stimulation, the image became much clearer than at any other rate. In a subsequent paper, details will be given of apparatus which was used to produce the image under carefully controlled conditions, and of methods which were adopted in a preliminary survey to determine the best rate of intermittent stimulation and the ratio of stimulus to rest most effective for the production of clear images.

Throughout the experiments colour changes were observed in the image after all but the shortest preliminary exposures. These changes were so clearly marked, and the image presented such an appearance of objective reality to some of the subjects who took part in the preliminary tests, that a systematic testing of a greater number of subjects was undertaken. In these experiments great individual differences were noted in the sequence of changes and also in the time of retention of the image. Over forty of these subjects had been previously tested for immediate visual memory, and on comparing their records for these memory tests with those obtained in connection with experiments to determine the time of retention of the image, it was noted that a marked relation existed between the two abilities under certain conditions.

It is hoped that the accounts of the work done will challenge a fuller investigation of the factors underlying the production of the projected image; and will suggest the utility of the method of intermittent stimulation in investigating certain visual phenomena.

\section{Method of Producing the Projected Image: Observations of its Chief Characteristics.}

The fact that a faded after-image can be revived upon renewed stimulation by weak, diffused light, has been frequently noted.

The following cases are typical instances:

McDougall 1 states: "If after fixating any patch of bright light on a dark background for thirty or sixty seconds, I completely exclude all light, and watch the after-image, it will disappear completely if all light be kept from the eyes, perhaps after two or three short periods of invisibility. If then a very little light be admitted to the retinæ for a few seconds (to expose the closed eyes to diffused daylight is often sufficient), the after-image will invariably recur for a short time and fade again after a few seconds when all light is excluded.

1 Mind, N.S. x. 55. 
If the original after-image be a bright one, it may be revived many times in this way and at each reappearance it is rather fainter than before, until it can be no longer revived by any means." Again, Titchener ${ }^{1}$ gives as an experiment: "Seat yourself before a brightly illuminated window which is partly covered by a shade and through which a gate, trellis-work, shrubs, etc., can be seen. Secure the head in a head-rest and fixate the window. Cover the eyes with the hands and keep them covered till there is no trace of the after-image in the dark field. Maintain the original fixation as nearly as you can. When the field is clear draw the hands rapidly aside without any jar to the head or eyes and bring them as rapidly together again.... The details of the scene, leaves of the shrub, string tassel of the window shade, etc., come out with surprising clearness."

In these experiments, the image produced by the admission of light gradually fades away. If, however, light be suddenly admitted a number of times in succession, by moving the hand or a screen between the source of light and the closed eyes, the image can be maintained for a considerable time, and each time the light is admitted there is a considerable increase in intensity of the image. It will also be noticed that a certain favourable rate of stimulation exists at which the successive images appear to fuse, and at times when the rate is successfully maintained the resulting image is remarkably clear.

A more striking example of the revival and maintenance of the image by intermittent stimulation, is seen in the following case. The eyes are directed and fixated for an instant on the disc of the sun when not too bright. On turning the eyes to another part of the sky, no image whatever may be seen if the exposure was short, but on blinking the eyes rapidly, a most distinct image, which is sometimes coloured, appears. This image can be projected in any direction by a suitable movement of the eyes, and on converging them the image can be brought from a distance to within a few feet of the eyes, where it remains so long as convergence is maintained, apparently in the air, with every appearance of objective reality. These marked changes which follow on the intermittent stimulation seem to indicate the need for a further investigation of the peculiarities of the image produced in this way.

The following experiments performed under bright adaptation and with foveal vision serve to bring out other peculiarities of the image and can be carried out under more convenient conditions. Though

\footnotetext{
1 Experimental Psychology, Students' Manual, 1. 28, Exp. 10 ; Instructors' Manual, 1. 46.
} Exp. 10. 
they are extremely simple, I believe that they point the way to more accurate work which should give a rich yield of facts in connection with the action of the retino-cerebral tract.

The simplest way of making these experiments is to sit with the back to a source of light and then fix the attention on a well illuminated sheet of paper placed at a distance of about two feet from the eyes. If now a filament of an electric bulb be fixated and a momentary flash be given, a distinct positive image will be seen on looking at the sheet of paper. This image dies away rapidly, but if the hand or a screen be moved rapidly between the eyes and the illuminated paper, a further series of bright after-images appears each time the paper is exposed. The images appear to equal the original in intensity, are much brighter than the ordinary after-image seen with closed eyes, and can be repeated for five or ten minutes according to the intensity and duration of the original exposure.

After prolonged exposure to light the resulting projected image is invariably coloured, and though the colour changes are best studied by using a bright uniformly illuminated disc as a stimulus, earlier experiments carried out with a bright filament present several points of interest. Though the sequence of colours has been found to vary in each individual, the following outline of the experiments dealing with short exposure first will give an idea of the character of the changes that take place.

Short exposure (about $0.5 \mathrm{sec}$. ): In this the filament image appears immediately and is apparently of equal intensity to the original. It gradually diminishes in intensity, reversals take place at irregular intervals till the final disappearance as a black or grey image. Throughout, the image is exceedingly clear and well defined in great contrast to the after-image proper. The black negative image is often indistinguishable from a black printed hair line.

Medium exposure (about 15 sec.): The filament appears almost immediately, but is rapidly tinged with red and appears as a hot wire. Later it is outlined in black or dark purple till at last this is further outlined in green. Finally, a vivid green image appears and alternates with the red; green invariably appearing last and sometimes of a very vivid hue.

Long exposure (60 sec. or more): For so long an exposure as this the eye is with difficulty kept steadily directed towards the filament and in the projected image it is often possible to trace the eye wanderings by a number of images slightly outside the main image. It is partly 
on this account that the image appears much coarser than in the previous cases.

At first no image of the filament can be seen, but the bright surrounding metal of the bulb and the reflections in the glass, appear as a fairly well defined image and present a distinctly purple or lilac colouring. In the course of time the filament appears, first faintly and surrounded by a dark outline and then after a time varying according to the length of the original exposure, increases in brightness till it appears to exceed that of the paper. If the exposure has been sufficiently long, the image appears distinctly yellow. The edges of the filament now begin to show a reddish tinge on the inner part, changing through purple to a faint green at the edge of the image. The whole image changes periodically to a dark purple or grey, and after each reversal the green outer margin increases in intensity and extent. In time the light green colour extends over the whole image, increases in intensity, and sometimes with a favourable rate of intermittency, which is in general slower than for the positive image, appears as an exceedingly vivid streak of green, and fading, finally vanishes. If the exposure has been very prolonged this final image is sometimes of a definite blue colour.

\section{The Part Played by Intermittent Stimulation.}

Throughout the literature dealing with after-images there is frequent reference to the projection of the negative after-images and the influence of the field upon which they are projected has been carefully noted. The case of the projected positive image has received less attention and the results obtained are somewhat conflicting, though it is generally accepted that the usual effect of a dark field for projection is to give a positive image, whereas, with a bright field, the image becomes negative. Whatever may be the final conclusion, the essential agreement that light reflected from the background upon which the image is projected, plays an important part in its formation, indicates the necessity for making a distinction at the outset between this image, which throughout this paper will be termed the 'projected image,' and the after-image proper, which is solely a result of continued activity in the visual tract.

The following experiments indicate the part played by the stimulation due to the illuminated screen and serve to bring out further differences between the projected image and the after-image proper.

A sheet of paper was prepared in the following manner. Strips of black paper were pasted in parallel rows at a distance apart of $8 \mathrm{~cm}$. 
and of a width of $4 \mathrm{~cm}$., and at right angles across these another set was fixed making a grid of black lines $4 \mathrm{~cm}$. wide with spaces of $8 \mathrm{~cm}$. By the side of these was pasted a square of black paper of equal area.

This screen was brightly illuminated, and was viewed through the openings of a cardboard disc, revolving at such a rate that the paper was exposed for about one-twentieth of a second as each opening passed. The white-hot filament was fixated for about two seconds, and the eye was directed to the grid seen through the revolving disc. The projected image appeared on the sheet and the filament spread right across the black lines of the grid ; and continued to do so for a considerable time till the image finally became negative. The image appeared quite as bright in those parts of the grid where there was but little reflected light as on the white parts of the screen. It was thought that there might perhaps be sufficient light from the black lines to give rise to the projected image over these. But in a subsequent test the eyes were turned to the black paper by the side of the grid. From this, however, no image could be obtained. Hence the formation of the complete image across the grid appeared to be due to other factors than direct stimulation of the previously excited area.

To test this question still more rigidly, a dark box $75 \mathrm{~cm} . \times 80 \mathrm{~cm}$. was prepared, with a circular aperture $8 \mathrm{~cm}$. in diameter and across this a ground glass screen was placed, having in the centre a tinfoil disc, blackened on the exposed side which faced the inside of the box. Behind the box was placed an incandescent burner, in front of this a lens and further, a revolving disc so arranged that light from the burner illuminated the ground glass disc practically simultaneously, as an adjustable aperture in the disc passed the light. The disc was revolved at a constant rate so that the illumination of the screen took place regularly at about the rate of eight per second. Black velvet was arranged in the box so that no reflected light entered the eye which was situated at a distance of $55 \mathrm{~cm}$. from the screen. The observer wore a black velvet mask and viewed the screen through two small blackened apertures so that as far as possible no light but what came directly from the screen entered the eyes. The observer now fixated a bright disc illuminated by light from an incandescent burner, transmitted through ground glass for five seconds. The disc was $7 \mathrm{~cm}$. in diameter, and placed at a distance of $55 \mathrm{~cm}$. Immediately afterwards the observer placed his head in the dark box and having fixated the black disc seen dimly on the screen, was covered with a cloth so as to completely exclude light from the box. An after-image appeared and faded. A shutter which 
had prevented light from falling on the screen of the dark box was now removed and immediately the first few flashes appeared, no black disc could be seen on the intermittently illuminated screen of the dark box. In its place was a disc, brighter than the surrounding screen and much brighter than the after-image previously seen. In a few seconds the image moved slightly and the black disc appeared as it were from behind the bright disc.

The image of the black disc on the screen would completely cover that part of the retina previously stimulated by exposure to the light of the bright disc. Hence this part was completely screened from the action of the intermittent light. The remainder of the retina was, however, fully exposed to the intermittent light from the screen and gave rise to the appearance of a bright disc in the middle of the uniformly illuminated screen and formed over the opaque black disc. This experiment in conjunction with a number of previous experiments made under less rigid conditions seems to show that the intermittent stimulation of the whole retina plays a part in the arousal of the projected image, and that the image can be formed in the absence of direct stimulation if the neighbouring parts of the retina be stimulated.

In order to test whether a reduction of the extent of the retinal stimulation would affect the character of the image, triangular strips of black paper, $1 \mathrm{~cm}$. wide at their base, $15 \mathrm{~cm}$. long and narrowing down to a point, were placed with their bases in a line so as to form a comb or series of gradually narrowing slits in which, when viewed by intermittent transmitted light, the bright area diminished towards the base. On looking at this after a flash from the filament, it was noticed that the image of the filament appeared as if behind the black lines when it was near the base, but appeared to cross them when the attention was directed to a point higher up. On an average it appeared to cross only where the illuminated surface was at least one-third of the total area, at a distance of about $5 \mathrm{~cm}$. from the base of the comb.

This last experiment, in conjunction with others of a similar type, seems to point to the fact that the conditions for producing the brightest projected image require the stimulation of a considerable portion of the retina, and the fact that the image can be obtained when there is no appreciable direct stimulation of the retinal area originally excited, as in the experiments with the grid, comb, and disc, points to the fact that the whole retina is concerned in the formation of the projected image.

If the image obtained after a long exposure be projected on a screen 
which has black strips of paper arranged like the comb previously mentioned, the image appears to run down to the base of the comb where there is only slight illumination and is with difficulty brought back to the brightly illuminated part of the screen. It seems as if the nerve energy, discharging readily into motor paths, reflexly brought about the turning of the injured part away from the action of the intermittent light.

The black lines of the comb appear in this case to prevent the spread of the image and the image appears as if entirely behind the comb. If, however, the image be very carefully fixated, the light part can be seen to spread gradually across the black bar from the bright wings of the image. This part, generally of much less intensity, frequently appears as of a red colour.

With prolonged exposure to an intense light the fatigue of the whole system prevents the appearance of any image. A small black dot placed as a fixation point, in the centre of the screen is invisible when an attempt is made to fixate it, yet on turning the eyes to any other part of the screen no dark patch appears. It seems as if a foveally situated 'blind spot' had been produced which, though not showing as black in the absence of sensation, is yet incapable of assisting in sensing the black fixation point. When, however, at a later stage a dark image appears, the black spot can be distinctly seen through the dark image.

This in conjunction with previous experiments seems to point to fatigue at higher levels on exposure to intense light in addition to retinal fatigue. It has been shown previously that the projected image can be produced without direct stimulation of the originally excited retinal area. If in the last mentioned experiment mere fatigue of the retinal area were present, there would have been sufficient 'spread' of impulse from neighbouring areas to have given rise to the image. The gradual appearance of the image would therefore seem to be due to the gradual recovery of the fatigued tracts.

The foregoing experiments seem to bring out the following facts. 'The 'projected image' noticed on first directing the eyes to an illuminated surface, appears to owe its revival to the restimulation of previously excited tracts and is also influenced by the excitation of the remainder of the retina and more particularly by the parts bordering on the previously excited area. The influence of this excitation is most effective in the initial stages and its effect rapidly dies away. Intermittent excitation causes a constant succession of these changes and 
when given at a suitable rate, results in the fusion of the most effective phases with the result that the projected image produced by intermittent stimulation shows a marked gain in intensity. Although these additional factors may justify a differentiation between the projected image and the after-image, from a physiological point of view, a mere increase of intensity alone is insufficient ground for a psychological distinction. The following observations, however, will indicate further points of difference and deal more particularly with the main psychological differences noted in the 'projection' of the image.

\section{Observations Concerning the Projection of the Image.}

Certain fundamental differences are noted during the course of the projected image when compared with the normal after-image. The contrast is best seen in the foveal image obtained by fixating a bright filament for about $0.5 \mathrm{sec}$. An exposure is given, and the eyes are covered. The after-image attains maximum brightness and fades. The outlines become indistinct. If at this stage stimuli be given by allowing light to fall intermittently on the closed eyelids, the clear definite outline can be seen developing as it were out of the confusion and the image appears as if situated at a definite distance from the observer, in marked contrast to the indefinite spatial relationship of the fading after-image. The effect is even more definite if the eyes be opened and directed to a screen during stimulation. From the outset, then, the phenomena presented by the two types of image exhibit distinct differences, and whereas the after-image appears to be located mainly by the help of memory images of the position of the original object, the projected image gives to consciousness, at the instant that it is formed, a definite awareness of spatial relationship.

Other factors in connection with the projection of the image appear to be of interest. The projected image is readily placed in any desired direction by a movement of the eyes. A large image can be projected on a bright cloud, and by passing the hand rapidly before the eyes and at the same time making an effort of convergence, the image can be brought from this distance to within a few inches of the eyes, diminishing in size and giving by its apparent movement through space a weird appearance of objective reality, somewhat similar to that ascribed to hallucinatory images. The distance seems to be entirely determined by the extent of convergence, and does not seem to be dependent on the 
presence of external objects other than the extent to which they give light for stimulation and serve to determine accommodation.

Though the image readily follows voluntary movements of the eye carried out in the usual way by the eye muscles, the following experiments seem to indicate the presence of other factors.

If a bright filament, or a small bright disc be fixated with both eyes and then the image be projected and kept constant by intermittent stimulation at a favourable rate, the deflection of one eyeball by slight side pressure does not double the image.

If when the eyeball is displaced, the two images of a fixation cross on the screen are kept in a constant relative position by carefully maintaining the same degree of displacement, the projected image can be made to travel from one cross to the other, and in fact can be made to occupy any position whatever with regard to the two crosses.

There is, of course, the possibility that one of the images might have been inhibited owing to the displacement or to some other cause and only the image of the undeflected eye might have been formed. But the following experiment negatives this.

A bright filament placed at an angle of $45^{\circ}$ from the vertical is illuminated and fixated with the right eye, the left being closed. Immediately afterwards, the left eye fixates the filament which has been turned through $90^{\circ}$. On stimulating the two eyes intermittently, the two images are seen, and form, combined, a cross: one stroke for the left eye and the other for the right eye. On displacing either eye the cross remains as perfect as before, showing that both eyes are forming the image and that there is neither inhibition nor displacement. In fact, both eyes may be quite considerably and actively displaced, making the spots on the screen move in quite a lively fashion, but the image of the cross remains fixed in whatever position the attention is directed.

- To test this more severely, shutters were placed in front of each eye, so arranged that they opened and shut alternately and were completely under the control of the operator. When they opened and shut slowly, the image of the cross appeared to be rocking, as first one part and then the other formed in the image. The speed of the shutters was increased, till at the rate of about four exposures per second, the image ceased to rock and appeared as a stationary cross. If when this point was reached one eye was displaced so that a spot on the screen was doubled, the cross remained as perfect as before the displacement, showing that although the direction of the visual axis of one eye had 
changed, the direction of projection of the image for that eye had not changed.

At first sight this seems to be explained by the fact that the retina of the left eye has received a stimulus on the corresponding area to that of the retina of the right eye, so that no matter how disparately the eyes are turned, the two images will coincide owing to the fact that the intermittent light stimulates both corresponding areas.

In normal conditions the image always follows the voluntary movement of the eye; whereas when the eyeball is displaced by pressure, the image no longer follows the involuntarily produced movements of the eye, but occupies a position which seems to be located by the voluntarily determined direction of attention. That the retina has moved is shown by the displacement of the spot on the screen, and the fact that in one case the image moves with the eye when the movement is voluntary, whereas when the movement is involuntary no displacement takes place, requires explanation.

There seems to be some connection between this phenomenon and the facts noted in the case of those individuals who are subject to auto-kinetic sensations. In those persons who are liable to eye movement in the dark, an after-image produced by previous fixation of a bright object remains apparently fixed, while a bright point of light fixated in the dark appears to move. In the case of others the image and the point of light seem to move together. In view of these individual differences it is possible that in respect to the projected image similar divergences may be found both with regard to the movement of the image on fixation and to the behaviour of the image on displacement of the eyeball. So far, however, I have not had the opportunity to test this.

In connection with the foregoing experiments, it is interesting to recall the work of Binet concerning the experimental study of hallucinations induced in hypnotic patients. He states that in every case examined, the hallucinatory image is doubled by pressure on one eyeball. On the other hand, Dr Hack Tuke ${ }^{1}$ and James ${ }^{2}$ give several examples of patients who do not respond to this test and in whom there is no doubling of the hallucinatory image on displacement of one eyeball, indicating that for this type of hallucination there is probably a central origin, and that this disturbance is projected.

The projected image under favourable circumstances is of a wellmarked hallucinatory character. When of considerable extent, it

1 Brain, XI.

2 Principles of Psychology, Ir. 136. 
sometimes becomes so real that at times the whole aspect of consciousness changes in a manner very similar to that noted in a certain type of hallucination ${ }^{1}$, and further, the agreement with regard to the doubling of the image on displacement of the eyeball gives additional grounds for hoping that with further enquiry an actual connection may be established between the phenomena of the projected image and certain types of hallucinations probably centrally excited.

\section{Suggested Explanations.}

The phenomena noted in the previous section are due to extremely complicated conditions and the following notes are added as suggestions of possibilities rather than as explanations.

At the outset, the action of intermittent stimulation calls for solution. Recent researches ${ }^{2}$ have shown that a momentary light stimulus is sensed as a series of pulses of gradually decreasing intensity. It is evident that the intermittent play of light on the retina will be instrumental in the formation of a succession of such series. The observation that there is a certain favourable rate of stimulation at which the projected image attains its maximum intensity seems to point to the fact that when this stage is reached there is an advantageous summation or reinforcement of pulses which, following rapidly, give rise to a steady continuous image. The fact that maximum effect is reached in those paths which have been recently stimulated, thus differentiating these, seems to imply "facilitation" of impulses along these tracts. In addition experimental evidence was advanced" ${ }^{3}$ which shows that the image can be revived by stimulation of neighbouring areas, in the absence of direct stimulation of the initially excited retinal areas. This indicates the possibility of a 'spread' of impulses from the rest of the retina and in particular from those paths bordering those originally stimulated. Finally the repeated light stimulus may also produce further changes in the visual substances which have been acted upon previously, leading to an increase in the intensity of the impulses in those tracts initially stimulated by these changes.

The marked difference between the after-image and the projected image in the case of short exposures seems to involve important features. The after-image being dependent entirely on the continued activity in

1 James, Principles of Psychology, Ir. 120.

a McDougall, this Journal, 1904, I. 93: "Momentary Stimulation of the Eye." See also Semon, Die mnemischen Empfindungen, 118.

- See Section III above. 
the visual tracts soon becomes blurred and indistinct, and it has been suggested that this change is due to the diffusion of substances formed during the initial stimulation and capable of exciting the retinal endings of the optic nerve. The marked way in which an indefinite after-image may be cleared up by intermittent stimulation through the closed eyelids and the fact that the projected image maintains its clearness of outline almost to its complete disappearance seem to show that the projected image depends mainly upon the re-excitation of the previously stimulated visual tracts by newly formed impulses, and to a lesser degree upon the re-excitation of the previously changed retinal substances. With longer exposures, and where the fixation is not accurately maintained, this difference between the two types of images is necessarily not so marked.

When the image is projected on a screen the adjustment of the eye muscles necessary to bring the fixation point of the screen to a focus involves the activity of definite efferent neural paths. The voluntary innervation of the muscles of convergence and the accompanying accommodation gives a partial neural disposition. The intermittent stimulations bring about the completion of neural conditions and activity similar to what would have been present if an actual object situated at the distance for which the eyes have been adjusted, were observed. These conditions, before the arrival of the efferent impulse, are, so to speak, only in a state of preparedness. On stimulation, the image is therefore seen 'projected' about the point of regard.

When, by suitably directing the eyes, the image is made to move amongst external objects, other factors are introduced. The problem noted in the experiments on the displacement of the eyeball is even more complicated and appears to involve the subject's total relational experiences with reference to the external world. It has been shown that an intended but unsuccessful contraction of an eye muscle produces apparent changes in the relationship of the external world with regard to the subject. Under normal conditions the successful voluntary contraction of the eye muscles produces no change in the relation of the outer world towards the subject but is experienced as an alteration of the attitude of the person towards external objects. The point of regard is changed, and with it the position of any projected image changes. From previous considerations it will be seen that the displacement of the eyeball by pressure, though causing changes in optical convergence, does not alter the willed coordinated muscular contractions. Hence no displacement of the image occurs. With movements of the 
eyes, images of stationary objects pass across the retina and in some cases pass across the part stimulated in the initial exposure. During voluntary movement of the eye these changes of stimulation are interpreted as a movement of the image amongst the stationary objects. When the eyeball is displaced by pressure, similar retinal changes take place, but the passage of the images of external objects is experienced, as is generally the case in the absence of willed movement, as a movement of the external objects. The projected image in this case, however, having its foundation, so to speak, in conditions which are unaltered, owing to the unchanged volitional attitude of the individual in this respect, remains stationary. The external objects apparently move about the image.

(Manuscript received 27 September, 1914.) 